\title{
DOENÇAS INFLAMATÓRIAS INTESTINAIS: CONSIDERAÇÕES FISIOLÓGICAS E ALTERNATIVAS TERAPÊUTICAS
}

\author{
Mônica de Oliveira Belém ${ }^{1}$ \\ Juliano Yasuo Oda ${ }^{2}$
}

BELÉM, M. de O.; ODA. J. Y. Doenças inflamatórias intestinais: considerações fisiológicas e alternativas terapêuticas Arq. Ciênc. Saúde UNIPAR, Umuarama, v. 19, n. 1, p. 73-79, jan./abr. 2014.

\begin{abstract}
RESUMO: Com o advento da industrialização e a mudança nos hábitos de vida mundial foi possível observar uma tendência para o surgimento de doenças, que passaram de maioria infecciosa para maioria inflamatória. Com o trato gastrointestinal não seria diferente, assim as doenças inflamatórias intestinais (DII) se tornaram mais comuns no mundo atual, sendo ainda mais presentes em países desenvolvidos e em desenvolvimento. Dentre as DII mais importantes destacam-se a Doença de Cronh (DC) e a Retocolite Ulcerativa (RCU), ambas de patogenia desconhecida, sem cura, sem padrão ouro para diagnóstico e que levam a pré-disposição ao câncer coloretal. Sabe-se apenas que o tabagismo, uso de contraceptivos orais, anti-inflamatórios não esteroidais, dieta, status social e geográfico, stress, flora entérica, alterações na permeabilidade intestinal e apendicectomia são fatores que predispõe ao surgimento dessas doenças. Ambas DII apresentam os sintomas de febre, dores abdominais, sangramento do cólon, diarreia e grave perda de peso. Sabe-se também que resultam de uma resposta imune inadequada em indivíduos geneticamente suscetíveis, aliado a complexas interações com fatores ambientais, microbianos e do sistema imune entérico. No Brasil não existe um levantamento do número de pacientes acometidos pelas DII. Isso aliado a ausência de um padrão ouro de diagnóstico para essas doenças leva a crer que há uma subestimação da quantidade real de casos. Diante de tantas incertezas esse artigo de revisão tem por objetivo apresentar as principais diferenças entre a DC e a RCU e mostrar os principais tratamentos existentes hoje.
\end{abstract}

PALAVRAS-CHAVE: Doenças inflamatórias intestinais; Tratamentos; Fatores biológicos; Doença de Cronh; Retocolite ulcerativa.

\section{INFLAMMATORY BOWEL DISEASES: PHYSIOLOGICAL CONSIDERATIONS AND ALTERNATIVE THERAPIES}

ABSTRACT: With industrialization and the change in global lifestyle, a tendency for the emergence of diseases can be observed, with changes from mostly infectious to mostly inflammatory. With the gastrointestinal tract it is no different. Therefore, inflammatory bowel diseases (IBD) have become more common in today's world, being even more present in developed and developing countries. Among the most important IBD there are the Cronh's disease (CD) and ulcerative colitis (UC), both of unknown pathogenesis, with no cure, no gold standard for diagnosis, and leading to predisposition for colorectal cancer. It is only known that smoking, the use of oral contraceptives, non-steroidal anti-inflammatory drugs, diet, social and geographical status, stress, enteric flora, changes in intestinal permeability and appendectomy are factors that predispose to the emergence of these diseases. Both IBDs present symptoms of fever, abdominal pain, bowel bleeding, diarrhea and severe weight loss. It is also known that they result from an inappropriate immune response in genetically susceptible individuals, combined with complex interactions with environmental and microbial factors, as well as the enteric immune system. In Brazil, there is no survey on the number of patients affected by IBD. This, combined with the absence of a diagnostic gold standard for these diseases, suggests that there is an underestimation of the actual number of cases. With so many uncertainties, this review article aims to present the main differences between CD and UC and show the main existing treatments at the moment.

KEYWORDS: Inflammatory bowel disease; Treatment; Biological factors; Cronh's disease; Ulcerative colitis.

\section{Introdução}

O trato gastrointestinal (TGI) é constituído anatomicamente por cavidade oral, faringe, esôfago, estômago, intestino delgado, intestino grosso e ânus (NETTER, 2011). Todas essas regiões, com exceção da boca, são histologicamente semelhantes, uma vez que todas são formadas, do lúmem para a região externa, por túnica mucosa, túnica submucosa, camada muscular e serosa ou adventícia, dependendo da região em questão (JUNQUEIRA; CARNEIRO, 2013).

O tubo digestório conta também com um sistema nervoso próprio conhecido como Sistema Nervoso Entérico (SNE), que é uma complexa subdivisão do sistema nervoso periférico. Apresenta-se na forma de plexos ganglionados entre as túnicas circular e longitudinal da camada muscular, sendo denominado de plexo mioentérico ou de Auerbach, responsável principalmente pela regulação do relaxamento e contração da musculatura; e outro que se encontra na camada submucosa denominado de plexo submucoso ou de Meissner, que é responsável por controlar os sentidos do lúmen e regular o fluxo de sangue gastrointestinal, bem como controlar a função das células epiteliais e sua secreção (VILLANACCI et al., 2008; NEZAMI; SRINIVASAN, 2010; FURNESS, 2012) (Figura 1).

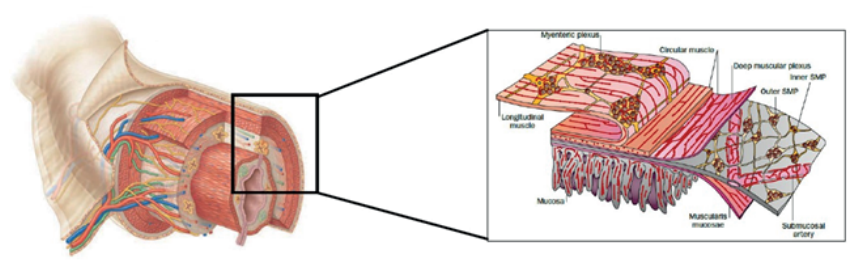

Figura 1: Esquema sobre a localização e morfologia do Sistema Nervoso Entérico (SNE)

${ }^{1}$ Biomédica, Mestranda do Programa de Pós Graduação em Patologia Experimental pela Universidade Estadual de Londrina (UEL); Laboratório de Neurogastroenterologia (UEL); monicabelem.uel@gmail.com (43) 3371-4600

${ }^{2}$ Fisioterapeuta, Mestre em Anatomia e Doutorando do Programa de Pós Graduação em Patologia Experimental pela Universidade Estadual de Londrina (UEL); Laboratório de Neurogastroenterologia (UEL) 
O TGI apresenta ainda bactérias que fazem parte da sua microbiota, estas não oferecem riscos de infecções se em homeostasia. Porém, o contato com bactérias e microrganismos presentes nos alimentos e na água podem desequilibrar essa homeostasia. Para proteção contra esses microrganismos o TGI conta com um tecido linfático associado à mucosa (GALT) que ocorre na forma de células imunes difusas presentes na lâmina própria das vilosidades da mucosa, bem como linfócitos arranjados em nódulos linfáticos, as Placas de Peyer (SILVERTHORN, 2010).

O TGI pode ser acometido por inflamações que alteram sua estrutura e seu funcionamento. As doenças inflamatórias intestinais (DII) apresentam-se principalmente como Doença de Cronh (DC) e Retocolite Ulcerativa (RCU), que são doenças crônicas recorrentes caracterizadas por dor abdominal, febre, sangramento retal, diarreia e graves perdas de peso, além de serem fator de risco para o câncer colo-retal (HENDRICKSON; GOKHALE; CHO, 2002; BAUMGART, 2009; ZHU; LI, 2012).

Tem-se observado que após a Segunda Guerra Mundial, devido a alterações nos hábitos de vida, a maior exposição a microrganismos, deficiência da resposta imunológica e higiene inadequada, uma tendência de mudança das doenças de infecciosas para inflamatórias crônicas e neoplásicas, o que aumenta a morbidade e a mortalidade (FIOCCHI, 2005; STEFANELLI et al., 2008; BAUMGART, 2009), estando as DII inclusas nesse novo grupo.

A patogenia das DII ainda é desconhecida, porém sabe-se que resulta de uma resposta imune inadequada em indivíduos geneticamente suscetíveis, aliado a complexas interações com fatores ambientais, microbianos e do sistema imune entérico (FIOCCHI, 2005; STEFANELLI et al., 2008). O não conhecimento da patogenia da doença contribui para que muitas opções terapêuticas tornem-se ineficazes na busca da cura para a condição da doença.

Pacientes com DII apresentam várias anormalidades estruturais e funcionais no sistema nervoso entérico. Porém, ainda não há uma concordância sobre o surgimento dessas alterações, pois não se sabe ainda ao certo se essas anormalidades são secundárias à inflamação (VILLANACCI et al., 2008), ou se a alteração neuronal, com aumento ou redução da quantidade normal de neurônios entéricos estaria relacionada com uma maior predisposição ao desenvolvimento da doença (MARGOLIS et al., 2011).

Mesmo não se conhecendo o motivo do surgimento das DII, sabe-se que alguns fatores de riscos aumentam a predisposição ao desenvolvimento dessas, como o tabagismo, dieta, status social e geográfico, stress, flora entérica, alterações na permeabilidade intestinal, apendicectomia, contracepção oral e utilização de anti-inflamatórios não esteroidais (FIOCCHI, 2005; STEFANELLI et al., 2008).

Não se sabe ao certo o número de casos reais das doenças inflamatórias intestinais, já que não existe um padrão ouro de diagnóstico, ficando esse restrito a exames radiológicos, endoscópicos e histopatológicos (BAUMGART, 2009). A faixa etária mais susceptível compreende de dez a quarenta anos. Não há predominância de sexo, mas uma possível associação com grupos étnicos específicos, pois a incidência é maior em caucasianos do que em negros e outras etnias (KARLINGER et al., 2000; PEARSON, 2004). Em 2012, nos Estados Unidos, o número estimado de pacientes com DII era de 1,4 milhões e de 30.000 novos casos diagnosticados por ano (ZHU; LI, 2012). Na Alemanha, em 2009, o número de pacientes diagnosticados com DII já chegava a 300.000 (BAUMGART, 2009). A ocorrência das DII é mais frequente na América do Norte, regiões nordeste e oeste da Europa; menos comum na África do Sul, Austrália e regiões central e sul da Europa e rara na Ásia e África (KARLINGER et al., 2000). Frente a isso, sabe que a prevalência de casos de DII é maior em países desenvolvidos e em desenvolvimento, sendo que no passado essa prevalência era restrita a países desenvolvidos (HENDRICKSON; GOKHALE; CHO, 2002; ZHU; LI, 2012).

No Brasil não há um levantamento a respeito do número de pacientes diagnosticados com DII, porém é possível perceber a preocupação da comunidade científica com as DII devido ao aumento do número de trabalhos epidemiológicos regionalizados como por exemplo GABURRI et al., (1998) em Juiz de Fora (MG), SOUZA et al., (2002) em Ribeirão Preto (SP), DE SOUZA; BELASCO; AGUILAR-NASCIMENTO, (2008) em diferentes municípios do Mato Grosso, OLIVEIRA; EMERICK; SOARES, (2010) na região leste do estado de Minas Gerais, KLEINUBING-JÚNIOR et al., (2011) em Joinville (SC), que mostram o aumento no número de casos diagnosticados de DII.

Diante do apresentado essa revisão teve por objetivo demonstrar as diferenças entre as duas principais formas de doenças inflamatórias intestinais, bem como suas alternativas terapêuticas para o tratamento conhecidas.

\section{Desenvolvimento}

Para a elaboração deste artigo foi realizado um levantamento bibliográfico de artigos científicos presentes na base de dados PubMed (http://www.ncbi.nlm.nih.gov/ pubmed/) valendo-se das palavras chaves: gut, intestine, inflammatory bowel disease, ulcerative colitis, Crohn disease, epidemiology e treatment. Essas deveriam aparecer no título e/ou no abstract do artigo, arranjadas três a três.

\section{Doença de Cronh (DC)}

A doença de Crohn pode envolver qualquer parte do trato gastrointestinal, ou seja da orofaringe até a região perianal. A inflamação ocorre de forma difusa, na qual segmentos inflamados são intercalados por segmentos sadios. $\mathrm{O}$ processo inflamatório é, na maioria das vezes, transmural, estendendo-se da camada mucosa, passando pela submucosa, muscular e frequentemente estendendo-se através da serosa, resultando em intervalos de entre as inflamações podendo ocorrer a formação de fístula. Os achados histológicos incluem pequenas ulcerações superficiais cercada de placa Peyer (úlcera aftoide) e inflamação crônica focal estendendo para a submucosa podendo formar granulomas. A localização mais frequente é na região ileocecal, seguida da região íleo terminal, difusa pelo intestino delgado, ou doença colônica isolada (HENDRICKSON; GOKHALE; CHO, 2002).

É caracterizada por apresentar sintomas mais sutis que a RCU, e por isso pode ter o diagnóstico tardio. Geralmente, estão associados ao desconforto gastrointestinal que envolve dores abdominais, diarreria, vômito, sangramento colônico, fissuras anais, distensão abdominal, perda de 
peso, saciedade precoce até estenose (HENDRICKSON; GOKHALE; CHO, 2002; NAYAR; RHODES; 2004). Esses sintomas dependem da localização, extensão e gravidade da inflamação como mostrado na Quadro 1.

Quadro 1: Principais sintomas da Doença de Cronh

\begin{tabular}{|c|c|}
\hline $\begin{array}{l}\text { Subtipo de } \\
\text { Doença de } \\
\text { Cronh }\end{array}$ & Principais Sintomas \\
\hline $\begin{array}{l}\text { Inflamação } \\
\text { restrita ao íleo }\end{array}$ & $\begin{array}{l}\text { Dor abdominal pós-prandial localizada } \\
\text { na área periumbilical, especialmente } \\
\text { em crianças, além de cólica abdomi- } \\
\text { nal crônica e diarreia o que dificulta a } \\
\text { distinção da síndrome do intestino ir- } \\
\text { ritável, aumento de proteína C reativa } \\
\text { sanguínea. }\end{array}$ \\
\hline $\begin{array}{l}\text { Inflamações } \\
\text { Gastroduodenais }\end{array}$ & $\begin{array}{l}\text { Saciedade precoce, náuseas, vômitos, } \\
\text { dor epigástrica, ou disfagia, devido à } \\
\text { dor pós-prandial e retardo no esvazia- } \\
\text { mento gástrico. }\end{array}$ \\
\hline $\begin{array}{l}\text { Pequena } \\
\text { extensão do } \\
\text { intestino }\end{array}$ & $\begin{array}{l}\text { Dor abdominal difusa, anorexia, diar- } \\
\text { reia e peso perda e pode resultar em má } \\
\text { absorção de lactose com sensibilidade } \\
\text { abdominal difusa. }\end{array}$ \\
\hline Colônica & $\begin{array}{l}\text { Pode imitar a retocolite ulcerativa, } \\
\text { apresentando diarreia com sangue e } \\
\text { muco associado com dor abdominal } \\
\text { inferior. }\end{array}$ \\
\hline Perianal & $\begin{array}{l}\text { Verrugas anais, fissuras anais e fístulas } \\
\text { com aumento das cólicas e distensão } \\
\text { abdominal e vômitos, pode evoluir para } \\
\text { estenose localizada com obstrução par- } \\
\text { cial ou completa em casos mais graves }\end{array}$ \\
\hline
\end{tabular}

A classificação da DC é feita de acordo com severidade, extensão e desenvolvimento da doença baseada no Índice de Atividade da Doença de Cronh (CDAI) (BEST et al., 1976), que é mais usado para estudos clínicos e avalia dor abdominal, bem-estar do paciente, número de sintomas extraintestinais associados (artralgia, artrite, inflamação da íris, uveíte, eritema nodoso, aftas orais, fissura anal, fístulas ou abscessos, outros tipos de fístulas e febre), ao uso de drogas antidiarreicas, massa abdominal, hematócrito e peso. Porém, para a prática clínica fatores como localização, extensão, desenvolvimento, idade, manifestações extraintestinais, histórico de vida do paciente e anamnese já auxiliam no diagnóstico (BRAZILIAN STUDY GROUP OF INFLAMMATORY BOWEL DISEASES, 2010).

Existem três níveis de DC: 1. Leve a moderada: paciente tolera alimentação enteral sem desidratação, toxicidade, desconforto abdominal, massa dolorosa, obstrução ou perda de peso maior que $10 \%$ da massa corporal; 2 . Moderada a grave: paciente não responde ao tratamento ou apresenta sintomas mais evidentes de febre, perda de peso, dor abdominal, náusea ou vômito intermitente (sem evidência de obstrução intestinal) ou anemia acentuada; 3. Severa para fulminante: paciente com sintomas persistentes apesar do uso de corticosteroides e/ou terapia biológica ou indivíduos com febre, vômitos persistentes, evidências de obstrução do intestino, sinais de irritação peritoneal, caquexia ou evidências de abscessos (BRAZILIAN STUDY GROUP OF INFLAMMATORY BOWEL DISEASES, 2010).

É conhecido que o tabagismo é um fator de risco para as DII, porém este exerce papel oposto nas DC e na RCU, já que o hábito de fumar é um fator de risco para a $\mathrm{DC}$, já que aumenta da frequência de recidiva da doença e necessidade de cirurgia, e sua interrupção melhora o curso da doença (STEFANELLI et al., 2008).

As lesões das DII ocorrem preferencialmente em regiões do intestino com maior presença de bactérias, como a válvula íleo-cecal e o cólon, e que cirurgias para desvio do fluxo fecal reduz as chances de recidiva da DC (D'HAENS et al., 1998), além do que um equilíbrio da flora entérica atenua o processo inflamatório das DII (STEFANELLI et al., 2008).

Sobre o perfil imunológico da DC, esta apresenta resposta do tipo Th1, já que as células T CD4+ intestinais produzem grandes quantidades de INF- $\gamma$ e overexpression de fator de transcrição T-bet, característico de Th1 (NEURATH et al., 2002), enquanto que os macrófagos da mucosa intestinal produzem grandes quantidades de IL-12 e IL-18 (MONTELEONE et al., 1997).

\section{Retocolite Ulcerativa (RCU)}

A retocolite ulcerativa é caracterizada por uma resposta inflamatória e alterações morfológicas restritas ao cólon. O reto está envolvido em $95 \%$ dos pacientes, com variáveis graus de extensão proximal. $\mathrm{O}$ processo inflamatório fica limitada principalmente à mucosa, marcado por abcessos nas criptas com grau variável de ulceração, edema e hemorragia ao longo de toda a extensão do órgão, as fístulas sobretudo são dificilmente encontradas (NAYAR; RHODES; 2004). Os achados histológicos característicos são caracterizados por inflamação aguda e crônica da mucosa devido a presença de leucócitos polimorfonucleares e células mononucleares, abcessos nas criptas, distorção das glândulas mucosas, depleção das células caliciformes e não há formação de granulomas (HENDRICKSON; GOKHALE; CHO, 2002; NAYAR; RHODES; 2004).

A característica mais marcante da RCU é a presença de sangue e muco misturado com fezes, que diferencia a RCU da síndrome do intestino irritável, já que as cólicas no abdômen inferior mais intensas nas evacuações são comuns aos dois diagnósticos (HENDRICKSON; GOKHALE; CHO, 2002; NAYAR; RHODES; 2004). Deve-se atentar para que os sintomas não sejam confundidos com os de uma diarreia infecciosa por microrganismo como Salmonella, Campylobacter ou Shigella spp e disenteria amebiana (NAYAR; RHODES; 2004).

A classificação da RCU se dá de acordo com a extensão anatômica da inflamação que é delimitada por avaliação colonoscópica. Existem três tipos de RCU: 1. Colite U1cerativa Distal: pode ser do tipo proctite, caracterizada pela inflamação da mucosa do reto na extensão de $15 \mathrm{~cm}$ a partir do esfíncter anal; ou do tipo proctosigmoidite, caracterizada pela inflamação da mucosa com extensão de $25-30 \mathrm{~cm}$ do esfíncter anal. 2. Colite Ulcerativa do lado esquerdo: inflamação da mucosa até flexura esplênica (às vezes até cólon transverso distal). 3. Pancolite: Inflamação da mucosa até o cólon transverso proximal, pode afetar a totalidade do có- 
Ion (BRAZILIAN STUDY GROUP OF INFLAMMATORY BOWEL DISEASES, 2010).

Já para a classificação da severidade da RCU a avaliação clínica pode ser feita de acordo com o índice de Truelove e Witts, que é dividido em severidade moderada, média ou severa de acordo com número de evacuações por dia, presença de sangue vermelho-vivo nas fezes, temperatura (febre ou não), pulsação, taxa de hemoglobina no sangue e velocidade de hemossedimentação (BRAZILIAN STUDY GROUP OF INFLAMMATORY BOWEL DISEASES, 2010).

É conhecido que para pacientes com RCU cessar o hábito de fumar tende a aumentar o risco de desenvolvimento do processo inflamatório, sugerindo assim um papel protetor do fumo para o desenvolvimento da doença (STEFANELLI et al., 2008).

Estudo sugere que os anticorpos IgG1 contra uma proteína estrutural de colonócitos foram produzidos seletivamente em pacientes com RCU, mas não nos com $\mathrm{CD}$, e essas imunoglobulinas podem estar subjacentes a patogênese da doença (TAKAHASI; DAS; 1985). Porém, não se sabe ao certo como essa doença se inicia.

Sobre o perfil imunológico da RCU, ao contrário do que é observado na DC, é um tipo atípico de resposta Th2, já que as células T CD1d restritas a NK produzem grandes quantidades de IL-13 e as células da mucosa intestinal produzem IL-5 (FUSS et al., 2004).

\section{Tratamento}

Como se sabe as DII não apresentam ainda cura conhecida, apenas tratamentos paliativos que não apresentam efeitos completamente satisfatórios devido à grande quantidade de efeitos colaterais e secundários (STEFANELLI et al., 2008).

Antes da escolha do tratamento a ser adotado para as DII é importante levar em consideração a severidade da inflamação a partir de resultados de exames clínicos, laboratoriais, endoscópicos e colonoscópicos, avaliar o risco de perfuração intestinal em casos mais severos, e também a dependência a corticoides (BRAZILIAN STUDY GROUP OF INFLAMMATORY BOWEL DISEASES, 2010).

As drogas de escolha para ao tratamento das DII são os anti-inflamatórios não esteroidais (AINEs), os corticoides, os imunossupressores e as terapias biológicas. Nenhuma dessas classes medicamentosas apresenta efeito realmente satisfatório, pois a eficácia é duvidosa e há muita geração de efeitos colaterais. Esses tratamentos podem ser classificados como agentes biológicos, peptídeos ou proteínas recombinantes, anticorpos e ácidos nucleicos. Ou classificados pela funcionalidade como inibidores de citocinas pró-inflamatórias, citocinas anti-inflamatórios, inibidores de moléculas de adesão celular, inibidores da polarização de Th1, inibidores da ativação e proliferação de células T, fatores de crescimento, inibidor da MAPK (proteínas cinases ativadas por mitógenos), imunomoduladores, bloqueadores de células T (anti-CD3, anti-CD4, anti-proliferação de células T) (STEFANELLI et al., 2008; DEVLIN; PANACCIONE, 2009; PITHADIA; JAIN, 2011; AHLUWALIA, 2012; CHAPARRO, 2012). As principais classes medicamentosas com exemplo de fármacos, seus principais efeitos desejados e colaterais podem ser conferidos no Quadro 2.

Quadro 2: Principais classes medicamentosas para o tratamento das doenças inflamatórias intestinais

\begin{tabular}{|c|c|c|c|}
\hline $\begin{array}{c}\text { Classe terapêutica } \\
\text { e Exemplo }\end{array}$ & Efeito desejado & Reação adversa (efeito colateral) & $\begin{array}{l}\text { Autores que sustentam a } \\
\text { terapia }\end{array}$ \\
\hline $\begin{array}{l}\text { Derivados Salicíli- } \\
\text { cos - } \\
\text { Ex. Sulfassalazina } \\
(\mathrm{SSZ})^{*}\end{array}$ & $\begin{array}{l}\text { - } \downarrow \text { Citocinas pró-inflamató- } \\
\text { rias } \\
\text {-Inibição da produção de } \\
\text { prostaglandinas e leucotrie- } \\
\text { nos } \\
\text { - } \downarrow \text { Estresse oxidativo } \\
\text { - } \downarrow \text { NF-kB } \\
\text {-Proliferação celular } \\
\text {-Inibição de apoptose }\end{array}$ & $\begin{array}{l}\text {-Dose-dependente e relacionada com } \\
\text { níveis séricos de sulfapiridina } \\
\text {-Dor abdominal, náuseas, vômitos, } \\
\text { anorexia, cefaleia, hemólise, inferti- } \\
\text { lidade masculina, hipersensibilidade, } \\
\text { alergia ou idiossincrasia, febre, exan- } \\
\text { tema, linfadenopatia, hepatite, pan- } \\
\text { creatite }\end{array}$ & $\begin{array}{l}\text { (NAYAR; RHODES, 2004; } \\
\text { DEVLIN; PANACCIONE, } \\
\text { 2009; BRAZILIAN STUDY } \\
\text { GROUP OF INFLAMMA- } \\
\text { TORY BOWEL DISEASES, } \\
\text { 2010; PITHADIA; JAIN, } \\
\text { 2011; CHAPARRO, 2012) }\end{array}$ \\
\hline $\begin{array}{l}\text { Corticosteroides } \\
\text { Ex. Hidrocortisona, } \\
\text { Prednisona, Predni- } \\
\text { solona e Budesoni- } \\
\text { da** }\end{array}$ & $\begin{array}{l}\text { - Usada para os casos mode- } \\
\text { rados e graves de DII } \\
\text { - Mais eficazes que os AINEs } \\
\text { no uso prolongado e em ene- } \\
\text { ma }\end{array}$ & $\begin{array}{l}\text {-Estimulação do apetite, aumento de } \\
\text { peso corporal, edema, insônia, la- } \\
\text { bilidade emocional, psicose, acne, } \\
\text { síndrome de Cushing, osteoporose, } \\
\text { osteonecrose, supressão da via hipo- } \\
\text { tálamo-hipófise-adrenal, infecções, } \\
\text { miopatias, catarata, atrofia da pele, } \\
\text { estrias, equimoses, esteatose hepáti- } \\
\text { ca, diabetes, hipertensão, glaucoma, } \\
\text { pancreatite aguda }\end{array}$ & $\begin{array}{l}\text { (SINGLETON et al., 1979; } \\
\text { MARSHALL; IRVINE, } \\
\text { 1997; HANAUER, et al., } \\
\text { 1998; NAYAR; RHODES, } \\
\text { 2004; DEVLIN; PANAC- } \\
\text { CIONE, 2009; BRAZILIAN } \\
\text { STUDY GROUP OF IN- } \\
\text { FLAMMATORY BOWEL } \\
\text { DISEASES, 2010; PITHA- } \\
\text { DIA; JAIN, 2011; CHAPAR- } \\
\text { RO, 2012) }\end{array}$ \\
\hline
\end{tabular}




\begin{tabular}{|c|c|c|c|}
\hline $\begin{array}{l}\text { Imunossupressores } \\
\text { Ex. Azatioprina } \\
\text { (AZA), 6-Mer- } \\
\text { captopurina (6- } \\
\text { MP), Metotrexato } \\
\text { (MTX) e Ciclos- } \\
\text { porina }\end{array}$ & $\begin{array}{l}\text {-Eficazes na remissão das } \\
\text { DII e para retirada dos corti- } \\
\text { coides em pacientes córtico- } \\
\text {-dependentes } \\
\text {-Para pacientes com resis- } \\
\text { tência, insensibilidade ou } \\
\text { dependência a corticoides } \\
\text {-Recidiva frequente das } \\
\text { crises após as retiradas dos } \\
\text { corticoides }\end{array}$ & $\begin{array}{l}\text { - } \uparrow \text { Suscetibilidade a doenças infeccio- } \\
\text { sas oportunistas, supressão da medu- } \\
\text { la óssea, leucopenia, mielotoxidade, } \\
\text { pancreatite aguda, náuseas, vômitos, } \\
\text { dores abdominais, febre, erupção } \\
\text { cutânea, mialgia, dores articulares, } \\
\text { parestesia, hipertensão arterial, hiper- } \\
\text { tricose, insuficiência renal } \\
\text {-Cefaleia } \\
\text {-Hiperplasia gengival } \\
\text {-Tonturas } \\
\text { Efeitos secundários desaparecem } \\
\text { com a descontinuidade de utilização } \\
\text { das drogas. }\end{array}$ & $\begin{array}{l}\text { (PEARSON et al., 2000; } \\
\text { HENDRICKSON; GOKHA- } \\
\text { LE; CHO, 2002; NAYAR; } \\
\text { RHODES, 2004; FIOCCHI, } \\
\text { 2005; ARDIZZONE et al., } \\
\text { 2006; GISBERT; GOMOL- } \\
\text { LÓN, 2008; STEFANELLI } \\
\text { et al., 2008; DEVLIN; PA- } \\
\text { NACCIONE, 2009; BRAZI- } \\
\text { LIAN STUDY GROUP OF } \\
\text { INFLAMMATORY BOWEL } \\
\text { DISEASES, 2010; PITHA- } \\
\text { DIA; JAIN, 2011; CHAPAR- } \\
\text { RO, 2012) }\end{array}$ \\
\hline
\end{tabular}

*Sulfassalazina (SSZ) é desdobrada no cólon pela enzima bacteriana em sulfapiridina azoredutase e ácido 5-aminossalicílico (5-ASA), o princípio ativo do medicamento. Pode ser administrado via oral ou retal, tópico.

**Budesonida é metabolizado rapidamente (aproximadamente $90 \%$ ) em produtos inativos pelo efeito de primeira passagem.

A terapia biológica é a tentativa de tratamento mais atual para as DII. Atua sobre mediadores e fenômenos naturais e fisiológicos. A utilização de anticorpo anti-TNF é a que mais se conhece. No Brasil há comercialização de Infliximab (anti-TNF, 75\% humano) e Adalimumab (anti-TNF, 100\% humano) (HENDRICKSON; GOKHALE; CHO, 2002; NAYAR; RHODES, 2004; BRAZILIAN STUDY GROUP OF INFLAMMATORY BOWEL DISEASES, 2010). Esse tipo de tratamento só é realizado se o paciente for insensível às terapias mencionadas anteriormente. Efeitos secundários anti-TNF ocorrem geralmente em menos de $10 \%$ dos casos (LICHTENSTEIN et al., 2006; FIDDER et al., 2009; CHAPARRO, 2012). Os efeitos colaterais mais comuns são reações à infusão, infecções do trato respiratório superior, bronquite, faringite, febre, cefaleia, náuseas, dor abdominal; com menos frequência: tonturas, dor torácica, atralgia, reações de hipersensibilidade retardada (abdominal ou perianal), pneumonia furunculose, obstrução intestinal, anemia hemolítica, disfunção cardíaca, lúpus induzido por drogas (anti-DNA positivo), e aumento do risco de linfoma (HENDRICKSON; GOKHALE; CHO, 2002; NAYAR; RHODES, 2004; BRAZILIAN STUDY GROUP OF INFLAMMATORY BOWEL DISEASES, 2010). O anti-TNF age ligando-se ao TNF solúvel circulante, impedindo-o de desempenhar o seu papel prÓ-inflamatório. Também neutraliza os receptores de TNF, o que resulta no bloco de sinalização da citocina, reduzindo assim o processo inflamatório. Além disso, o anti-TNF liga-se aos TNF ligado a produção de células de membrana (transmembrana do TNF) e produz sinalização reversa que inibe a produção de TNF e induz a apoptose de células produtoras de TNF. Quando anti-TNF liga-se a receptores transmembranares ou TNF facilita a ativação do complemento e a fagocitose de imunocomplexo (PEYRIN-BIROULET et al., 2008).

\section{Conclusão}

Diante do exposto nesse artigo é possível perceber que ainda é pouco o que se sabe sobre as principais doenças inflamatórias intestinais, desde sua patogênese, desenvolvimento, implicações aos pacientes e sobre tudo o tratamento, já que todos os tipos de terapias medicamentosas acarretam uma grande quantidade de efeitos colaterais indesejáveis que diminuem a qualidade de vida do paciente. Ainda há o risco de se confundir as DII com outros tipos de doenças devido a não existência de um padrão ouro para o diagnóstico dessas doenças, o que prejudica ainda mais o paciente, já que o correto tratamento é postergado. É importante também salientar a tendência ao desenvolvimento de câncer colo-retal por pacientes que apresentam alguma das formas das DII. Assim, sugere-se que novos estudos sejam realizados para melhor entendimento da Doença de Cronh e da Retocolite Ulcerativa e busca por novos e melhores direcionados tratamentos diante do estágio, severidade e perfil imunológico do paciente, a fim de que se possa melhorar a qualidade de vida desses.

\section{Referências}

ARDIZZONE, S. et al. Randomised controlled trial of azathioprine and 5-aminosalicylic acid for treatment of steroid dependent ulcerative colitis. Gut. v. 55, n. 1 , p. 47$55,2006$.

AHLUWALIA, J. P. Immunotherapy in Inflammatory Bowel Disease. Med Clin N Am. v. 96, n. 1, p. 525-544, 2012.

BAUMGART, D. C. The Diagnosis and Treatment of Crohn's Disease and Ulcerative Colitis. Deutsches Ärzteblatt Inter. v. 106, n. 8, p. 123-133, 2009.

BEST, W. R. et al. Development of a Crohn's disease activity index. National Cooperative Crohn's Disease Study. Gastroenterol. v. 70, n. 1, p. 439-44, 1976.

\section{BRAZILIAN STUDY GROUP OF INFLAMMATORY} BOWEL DISEASES. Consensus Guidelines For The Management Of Inflammatory Bowel Disease. Arq. Gastroenterol. v. 47, n. 3, p. 313-325, 2010.

CHAPARRO, M. Novedades sobre la eficacia, optimización y seguridade de los tratamientos de la enfermedad inflamatoria intestinal. Gastroenterol Hepatol. v. 35, n. 1, p. 57-67, 2012.

DEVLIN, S. M.; PANACCIONE, R. Evolving 
Inflammatory Bowel Disease Treatment Paradigms: TopDown Versus Step-Up. Gastroenterol Clin N Am. v. 38, n. 1, p. 577-594, 2009.

DE SOUZA, M. M.; BELASCO, A. G. S.; AGUILARNASCIMENTO, J. E. Perfil Epidemiológico dos Pacientes Portadores de Doença Inflamatória Intestinal do Estado de Mato Grosso. Rev bras Coloproct, v. 28, n. 3, p. 234-238, 2008.

D'HAENS, G. R. et al. Early lesions of recurrent Crohn's disease caused by infusion of intestinal contents in excluded ileum. Gastroenterol. v. 114, n. 1, p. 262-267, 1998.

FIDDER, H. et al. Long-term safety of infliximab for the treatment of inflammatory bowel disease: a single-centre cohort study. Gut. v. 58, n. 1, p. 501-8, 2009.

FIOCCHI, C. Inflammatory bowel disease pathogenesis: therapeutic implications. Chin. J. Dig. Diseases, v. 6, n. 1, p. 6-9, 2005.

FURNESS, J. B. The enteric nervous system and neurogastroenterology. Nat. Rev. Gastroenterol. Hepatol, v. 9 , n. 5, p. 286-294, 2012.

FUSS, I. J. et al. Nonclassical CD1d-restricted NK T cells that produce IL-13 characterize an atypical Th2 response in ulcerative colitis. J. Clin. Invest., v. 113, n. 1, p. 14901497, 2004.

GABURRI, P. D. et al. Epidemiologia, aspectos clínicos e evolutivos da doença de Crohn: estudo de 60 casos. Arq. Gastroenterol. v. 35, n. 1, p. 240-246, 1998.

GISBERT, J. P.; GOMOLLÓN, F. Thiopurine-induced myelotoxicity in patients with inflammatory bowel disease: a review. Am. J. Gastroenterol. v. 103, n. 1, p.1783-800, 2008.

HANAUER S. B. et al. Budesonide enema for the treatment of active, distal ulcerative colitis and proctitis: a doseranging study. Gastroenterol. v. 115, n. 1, p. 525-32, 1998.

HENDRICKSON, B. A.; GOKHALE, R.; CHO, J. H. Clinical Aspects and Pathophysiology of Inflammatory Bowel Disease. Clin. Microbiol. Rev. v. 15, n. 1, p. 79-94, 2002.

JUNQUEIRA, L. C.; CARNEIRO, J. Histologia Básica. Rio de Janeiro: Guanabara Koogan, 2013, 12. ed. 556 p.

KARLINGER, K. et al. The epidemiology and the pathogenesis of inflammatory bowel disease. Eur. J. Radiol. v. 35, n. 1, p. 154-167, 2000.

KLEINUBING-JÚNIOR, et al. Perfil dos pacientes ambulatoriais com doenças inflamatórias intestinais. Arq. Bras. Cir. Dig. v. 24, n. 3, p. 200-203, 2011.

LICHTENSTEIN, G. R. et al. Serious infections and mortality in association with therapies for Crohn's disease: TREAT registry. Clin. Gastroenterol. Hepatol. v. 4, n. 1, p. 621-30, 2006.

MARGOLIS, K. G. et al. Enteric neuronal density contributes to the severity of intestinal inflammation. Gastroenterol. v. 141, n. 1, p. 588-598, 2011.

MARSHALL, J. K.; IRVINE, E. J. Rectal corticosteroids versus alternative treatments in ulcerative colitis: a metaanalysis. Gut. v. 40, n. 1, 775-81, 1997.

MONTELEONE, G. et al. Interleukin 12 is expressed and actively released by Crohn's disease intestinal lamina propria mononuclear cells. Gastroenterol, v. 112, n. 1, p. 1169-1178, 1997.

NAYAR, M.; RHODES, J. M. Management of inflammatory bowel disease. Postgrad. Med. J. v. 80, n. 1, p. 206-213, 2004.

NETTER, F. H. Atlas de Anatomia Humana. Rio de Janeiro: Elsevier, 2011, 5. ed. 624 p.

NEURATH, M. F. et al. The Transcription Factor T-bet Regulates Mucosal T Cell Activation in Experimental Colitis and Crohn's Disease. J. Exp. Med. v. 195, n. 1, p. 1129-1143, 2002.

NEZAMI, B. G.; SRINIVASAN, S. Enteric nervous system in the small intestine: Pathohysiology and clinical implications. Curr. Gastroenterol. Rep. v. 12, n. 5, p. 358$365,2010$.

PEARSON, C. Inflammatory bowel disease. Nurs. Times. v. 100, n. 9, p. 86-90, 2004.

PEARSON, D. C. et al. Azathioprine for maintaining remission of Crohn's disease. Cochrane Database Syst Ver. v. 2, CD.00006, 2000.

PEYRIN-BIROULET, L. et al. Crohn's disease: beyond antagonists of tumour necrosis factor. Lancet. v. 372, n. 1, p. 67-81, 2008.

PITHADIA, A. B.; JAIN, S. Treatment of inflammatory bowel disease (IBD). Pharmacolol. Rep. v. 63, n. 1, p. 629642, 2011.

OLIVEIRA, F. M.; EMERICK, A. P. C.; SOARES, E. G. Aspectos epidemiológicos das doenças intestinais inflamatórias na macrorregião de saúde leste do Estado de Minas Gerais. Cien. Saude Colet. v. 15, n. 1, p. 1031-1037, 2010 .

SILVERTHORN, D. U. Fisiologia Humana: Uma abordagem integrada. Porto Alegre: Artmed, 2010, 5. ed. $992 \mathrm{p}$.

SINGLETON, J. W. et al. National Cooperative Crohn's Disease Study: adverse reactions to study drugs. 
Gastroenterol. v. 77, n. 1, p. 870-82, 1979.

SOUZA, M. H. L. P. et al. Evolução da ocorrência (19801999) da doença de Crohn e da retocolite ulcerativa idiopática e análise das suas características clínicas em um hospital universitário do Sudeste do Brasil. Arq.

Gastroenterol. v. 39, n. 2, p. 98-105, 2002.

STEFANELLI, T. et al. New Insights into Inflammatory Bowel Disease Pathophysiology: Paving the Way for Novel Therapeutic Targets. Curr. Drug Targets. v. 9, n. 5, p. 413$418,2008$.

TAKAHASI, F.; DAS, K. M. Isolation and characterization of a colonic autoantigen specifically recognized by colon tissuebound immunoglobulin $\mathrm{G}$ from idiopathic ulcerative colitis. J. Clin. Invest. v. 76, n. 1, p. 311-318, 1985.

VILLANACCI, V. et al. Enteric nervous system abnormalities in inflammatory bowel diseases.

Neurogastroenterol. Motil. v. 20, n. 9, p. 1009-1016, 2008.

ZHU, H.; LI, Y. R. Oxidative stress and redox signaling mechanisms of inflammatory bowel disease: updated experimental and clinical evidence. Exp. Biol. Med. v. 237, n. 5, p. 474-480, 2012. 